\title{
ERRATUM: Test tubes go microscopic
}

Daniel Evanko

Nat. Methods 2, 244-245 (2005).

In the version of this article originally published, page numbers for the second reference were listed incorrectly. The corrected reference should read:

Rondelez, Y. et al. Highly coupled ATP synthesis by F ${ }_{1}$-ATPase single molecules. Nature 433, 773-777 (2005b).

\section{CORRIGENDUM: In vivo protein biotinylation for identification of organ-specific antigens accessible from the vasculature}

Jascha-N Rybak, Anna Ettorre, Brigitte Kaissling, Raffaella Giavazzi, Dario Neri \& Giuliano Elia

Nat. Methods 2, 291-298 (2005).

In the version of this article originally published, an omission was made in the acknowledgments section. The revised paragraph is printed below.

The authors are grateful to M. Le Hir (Anatomy Institute, University of Zurich) for help in the vascular perfusion fixation of mice used in indirect immunofluorescence experiments, to A. Garofalo (Mario Negri Institute) for technical support and to the members of the Functional Genomics Center Zurich for granting access to the instrumentation and for their help. Financial support of the Gebert-Rüf Foundation, the Swiss National Science Foundation and the Bundesamt für Bildung und Wissenschaft (European Union Projects Angiogenesis and Stroma FPS/6 \#503233) is gratefully acknowledged. J.-N.R. is a recipient of a Bundesamt für Bildung und Wissenschaft salary (FLUOR-MMPI). G.E. is on leave of absence from the Institute of Neurobiology and Molecular Medicine CNR, Rome, Italy. 